\title{
Audit and Review of Ankylosing Spondylitis Management in Qatar
}

\author{
Abdul-Wahab Al-Allaf* and Yousef M Yahia \\ Rheumatology Section, Department of Medicine, Hamad Medical institute, Doha \\ *Corresponding author: Abdul-Wahab Al-Allaf, Rheumatology Section, Department of Medicine, Hamad Medical institute, \\ Doha
}

\section{ARTICLE INFO}

Received: 㓞 June 11, 2021

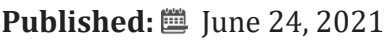

Citation: Abdul-Wahab Al-Allaf, Yousef M Yahia. Audit and Review of Ankylosing Spondylitis Management in Qatar. Biomed J Sci \& Tech Res 36(5)-2021. BJSTR. MS.ID.005904.

Keywords: Ankylosing Spondylitis; Audit; Management; Tuberculosis; BASDAI

\section{ABSTRACT}

Introduction: AS is a chronic inflammatory condition with a worldwide distribution. AS diagnosis and treatment are well standardized with international guidelines for its management. However, the application for these guidelines varies from country to country and even from Centre to Centre in the same country. Hence, we are auditing our AS service.

Method: Electronic medical records of AS patients attending the out-patient department for a full year were reviewed for various aspect of AS management.

Results: We audit 205 AS attended the out-patient during one year. $26 \%$ of our cohort found to have nr-asSpA with M: F ratio was 1:1 as compared to 4:1 in patients with positive X-ray. Patients' characteristics compared with other local and international studies. There were documentation issues. All our patients tried the NSAIDs as the first line and then moved to TNF-a biologics. $56 \%$ of our cohort were on biologics and $23 \%$ for those tested for latent tuberculosis, were positive and required treatment before starting the biologics. Only 7-10\% had their objective assessment of ASDAS \& BASDAI at various stage of their disease.

Conclusions: In a routine clinic scenario, there are major documentation issues and discrepancies between what we should do and what is done.

\section{Introduction}

Ankylosing spondylitis (AS) is a chronic inflammatory condition and it is one of the main Spondyloarthropathy ( $\mathrm{SpA}$ ) group with a worldwide distribution [1]. The incidence is between 0.5-14 per 100,000 people per year [2,3]. Sacroiliac joints (SIJ) are the main joints involved in such patients and in some peripheral joints could be involved as well [4]. The main extra-articular manifestations of AS include anterior uveitis [5]. In the majority of the cases, the diagnosis could be delayed for a few years from the onset of its symptoms [6]. A few studies were conducted on the characteristics of AS in the Middle East [7-10].

AS diagnosis and treatment are well standardized with clear international guidelines for its management. However, the application for these guidelines varies from country to country and even from Centre to Centre in the same country. Hence, we are auditing our AS service as compared to one of the main international criteria.

We can't find any auditing for how good we are doing for our AS patients locally in the middle east. We always find some discrepancy between the evidence-based guidelines and what is practically done with regards to various disease management including the AS. Accordingly, our primary outcome from this project is to audit how we are doing regarding AS management compared with the evidenced-based practical guidelines. We also studied some of the characteristics of AS patients in Qatar as a secondary outcome.

\section{Methods}

Electronic medical records of patients who visited Hamad General Hospital rheumatology clinic for 12 months from 1ST Jan to 
31ST December 2017 were reviewed. The Diagnosis of Ankylosing spondylitis is based on positive symptomatic and radiological findings. We looked for the various aspect for AS including its characteristics, diagnosis, service, assessment and management as compared to the 2016 update of the ASAS-EULAR management recommendations for axial spondyloarthritis as our benchmark for the comparison, which is one of the main evidence-based management recommendations criteria for axial spondyloarthritis [11].

\section{Criteria Audited are:}

a) Criterion 1: Documentations issues.

b) Criterion 2: Diagnosis (X-ray, MRI, HLA-B27).

c) Criterion 3: The use of NSAIDs (as a First-line therapy).

d) Criterion 4: Biologic use, which divided into 4 subgroups.

e) Criterion 4.1: Pre biology tests for Hep B and C, QuantiFERON / PPD.

f) Criterion 4.2: Vaccination (Flue \& Pneumococcal).

g) Criterion 4.3: Biology type used.

h) Criterion 4.4: Pre and Post biology assessment.

\section{Results}

A total of 205 AS patients were included during 2017. Males were $146(70 \%)$ with a male to female's ratio of 2.3:1. However, for those with positive X-ray the male to female ratio was 4:1 and for those with nr-as SPA, it was 1:1. The mean age was 41.5 years, and the mean age at diagnosis was 32 years, and the disease duration was 9.8 years. 117 (57\%) of our cohort were Arabs with 67 of them were Egyptian and 25 were Qatari. HLA-B27 results were documented in 129 (63\%) of our cohort and it was positive in $90(70 \%)$ of them. For those 90 with the positive HLA-B27, the distribution was $63 \%$ of the Arabic patients and $37 \%$ of the nonArabs. However, for those AS patients with uveitis, the HLA-B27 was tested in $27 / 38$ patients, and it was present in $23 / 27$ (85\%). $144(70 \%)$ has a SIJ X-Ray which was normal in 37 (26\%) patients, all of the homes had abnormal SIJ with clear sacroiliitis on the MRI scan; nr-axSpA. MRI scan was positive for sacroiliitis in $90 \%$ of the whole cohort. Uveitis was present in 38 (18\%), and peripheral joint involvement in $20 \%$.

For those with the nr-axSpA (26\%): they had a similar HLA-B27 positivity as in radiological positive patients (72\% vs $70 \%)$, but these patients had a much higher peripheral joints involvement which was $45 \%$ vs only $20 \%$ of the whole cohort and affects male and female similar with the male to female ratio of $1: 1$ vs $4: 1$ for those with positive X-ray sacroiliitis. We found that there is a lack of documentation for some of the important characteristics in our cohort. The family history of AS and SpA was documented in only
$14 \%$ and the age at diagnosis was documented in only $86(42 \%)$ patients and disease duration in only $66(32 \%)$ patients. The smoking status which is important in managing these patients was documented in only $48 \%$.

Correct Diagnosis was secured in all our patients based on ASAS criteria (100\%). All our AS patients have had a trial of two NSAIDs as per EULAR criteria (100\%) and at the time of the study, $66 \%$ of all our AS cohort continued on NSAIDs. The first biologics in all our patients was Anti-TNF-a as per guidelines criteria (100\%). Only $15 \%$ of all our AS patients were on DMARDs, mainly SSZ and $56 \%$ of those on DMARDs suffered from peripheral joints involvements. We found there was a significant problem with lack of the objective assessment of disease severity and activity in the majority of our patients in various stages of their disease (Table 1).

Table 1: Audit criteria achievement.

$\left.\begin{array}{|c|c|}\hline \text { Criteria } & \text { \% Achieved } \\ \hline \begin{array}{c}\text { C } 1 \text { Documentations of various characteristic } \\ \text { and outcome measures }\end{array} & \text { Range from 7-56\% } \\ \hline \text { C 2 Correct Diagnosis as per ASAS criteria } & 100 \% \\ \hline \text { C 3 NSAIDs used as initial therapy } & 100 \% \\ \hline \text { C 4 The first line biologic was TNF-a blockers } \\ \hline \text { C 4.1 The objective assessment of ASDAS \& } & 100 \% \\ \hline \text { C 4.2 Pre-biologic screening for TB \& Viral } \\ \text { Hepatitis }\end{array}\right)$

In total we founded 109 (53\%) of our cohort were on biologics with Adalimumab (43\%) was the most commonly used followed by Etanercept (26\%) (Figure 1). Of those on biologics, 33 (30\%) patients tried more than one biologic during their disease course. The most common reason for trying another biologic was related to the primary or secondary failure. Out of the 109 patients on biologics, tuberculosis screening was done/documented in only 61 $(56 \%)$ patients, and almost a quarter of them (23\%) were positive for latent tuberculosis and they received the required treatment (Figure 1).

Out of the 109 patients on biologics, hepatitis B and C viral screening was done/documented in only 61 (56\%) patients, and this was positive in only $5 \%$ and they received the required treatment. Pre-biologic vaccinations with the pneumococcal vaccine were given/documented in only 60/109 (55\%) patients and annual flu vaccine in 33/109 (30\%) patients. This could be a real figure, or it could be related to those in whom the vaccine has been documented. Initial BASDAI score was done/documented in only $10 \%$ at the time of diagnosis and in only $8 \%$ pre-biologic use and only $11 \%$ in post biologic use, for ASDAS these figures $4 \%, 6 \%$ and $6 \%$ prospectively. 


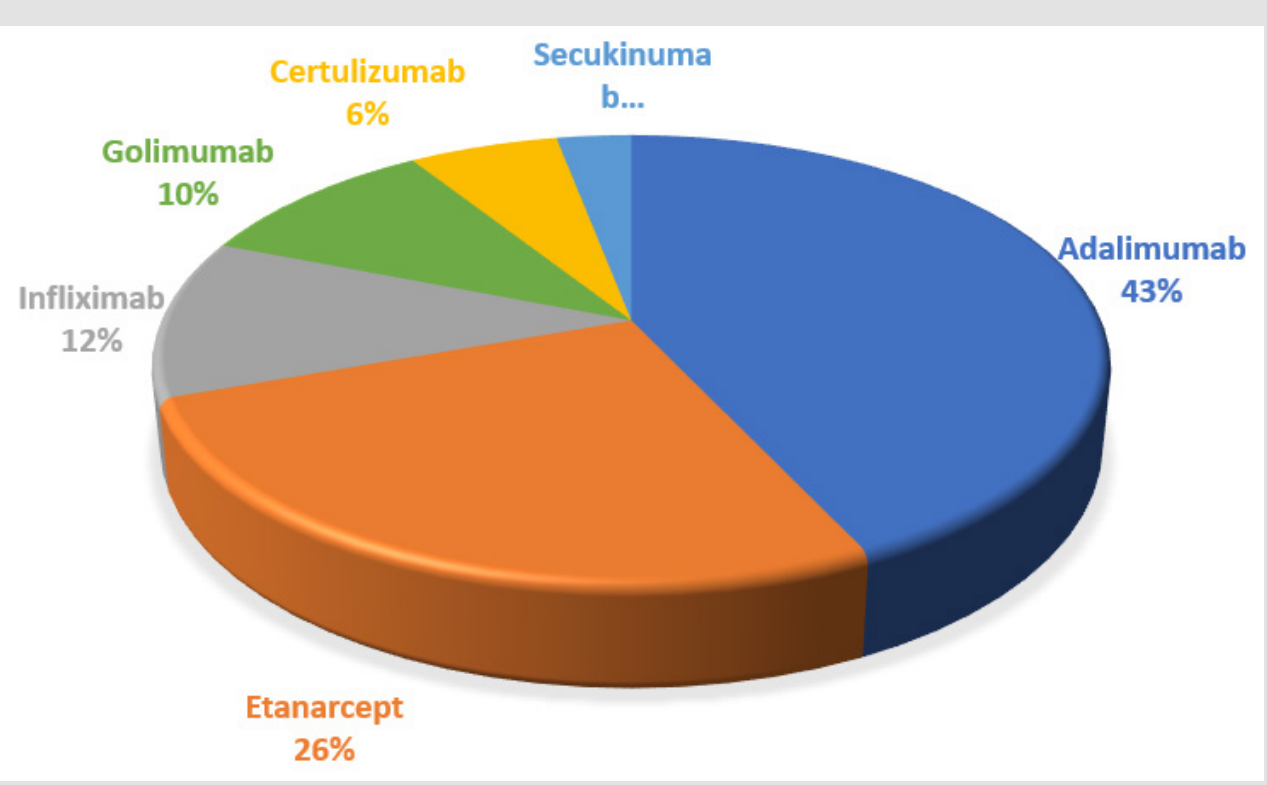

Figure 1: Distribution of biological agents used during 2017 (109 patients).

\section{Limitations}

There is a major documentation issue for various aspects of AS management. It is a retrospective study.

\section{Discussion}

Our AS patient's characteristic as compared to our department previous study in 2015 [10] had the same age mean age at diagnosis of 32 years, lower M: F ratio of 2.3:1 vs 5.2:1, more Arabs (57\% vs $40 \%$ ), less positivity of HLA-B27 (70\% vs $81 \%$ ). Both studies had the same DMARDs of $15 \%$ with the majority receiving SSZ, and NSAIDs use (66\% vs $69 \%$ ). However, our biological use increased over time (3 years) from $40 \%$ to $53 \%$ in our recent study. We have slightly more uveitis patients (18\% vs $14.5 \%)$ and much less peripheral arthritis (20\% vs $47 \%$ ) as compared with the previous study. Interestingly, and as expected, all the previous study AS patients had their BASDAI and BASFI assessment as it is part of the study protocol, but only $7 \%$ of our patients have BASDAI as part of routine clinic management.

Male predominance was observed in all the local and international studies. Our study male to female ratio was comparable to what has been found in Saudi Arabia (2.8:1) [8], and it was much lower than what has been found in Jordanian (8.1:1) [7]. For European and South American this ratio was 4.65:1 and $5.1: 1$, respectively $[12,13]$. Our peripheral joints involvement of $20 \%$ was lower as compared to other studies; $33 \%$ in Saudi Arabia [8], 34\% in Jordan [7], 36.2 in Moroccan [14]. Our Uveitis figures of $18 \%$ were slightly higher as compared with other studies of $13 \%$ in Jordanians [7], 14.3 in Brazilian [13]. Uveitis figure was even much lower in Moroccans at 6.8\% [14], but it was much higher (44.9\%) in Iranians [15]. In systematic metanalysis uveitis reported to be present in $25.8 \%$ which was slightly higher in North America and
Europe as compared to Asia and Latin America [16]. This difference in uveitis could be due to the differences in HLA-B27 prevalence in different areas.

HLA-B27 prevalence in our study of $70 \%$ was comparable to others in the region. In Saudi Arabia it was 68\% [8], in Jordan $72 \%$ \& 81\% [7,17] and in Kuwait 82.6\% [18]. However, HLA-B27 present in $90-95 \%$ of white western European AS as compared with $8 \%$ in their general population and the risk of AS developing is as high as 5\% in HLA-B27 positive individuals and substantially higher $20 \%$ in HLA-B27 positive relatives of patients. However, most of HLA-B27 positive induvial remain healthy $[19,20]$. There is a significant delay of diagnosis of 11.4 years in those who were HLA-B27 negative as compared with 8.5 years in HLA-B27 positive patients [21]. It is difficult to compare the use of TNF-a blockers and other treatment due to the difference in when these studies have been done.

In our cohort we have found that 109 (53\%) of our patients in our cohort were on biologics with Adalimumab was the most commonly used followed by Etanercept. In an audit in the UK, it has been found that $41 \%$ of their AS patients were on TNF-a blockers with Etanercept (51\%) has slightly higher figures than Adalimumab (45\%) and only $4 \%$ were on Infliximab [22]. The lower figures in the UK study could be explained by the restriction of biological use as per the NICE guidelines in the UK. I did a previous audit for AS patients who were on Biologics in the UK in 2010 which has been re-audited in 2013 (not published). The results from the 2013 audits were: the male to female ratio was 4:1 and HLA-B27 was present in 92\%. Two BASDAI readings were done 12 weeks apart before the start of biologics in $95 \%$ of the patient as this was mandatory to assess their eligibility for biologic as per our NICE guideline [NICE guidelines on Ankylosing Spondylitis 2008 and updated on 2010, www.nice.org.uk]. $75 \%$ of that cohort were on 
Adalimumab, $15 \%$ on Etanercept and $10 \%$ on Infliximab. Response assessment was done in $82.5 \%$ at 12 weeks of the treatment and the remaining was after that due to non-attendance to the clinic appointment. Response reported in $83.3 \%$ (reduction of BASDAI to $50 \%$ of pre-treatment value of by 2 or more units) with $75 \%$ has adequate response and 25\% inadequate response and all of them continued on the biologics and for those with primary failure, the biologics have been stopped.

When BASDAI or ASDAS is not mandatory, the uptake for these assessments was very poor routinely. We think it should be mandatory at least for those going to be on biologics for better objective assessment for response and it will guide us for amending the medications and possibly changing the biologic treatment. We feel that we need to improve our services for AS patients by better documentation for the necessary items such as history for smoking. These patients need good advising and referral for smoking cessation as an important aspect of managing these patients and which is very often missed.

Documentation issue could be helped by designing a progress note in a Prefilled electronic tick boxes format during each visit for AS and to be filled before starting biologic agent and subsequently on follow up.All our AS cases fulfil the ASAS diagnostic criteria. We achieved $100 \%$ with regards to the use of NSAIDs and biologics as compared to EULAR 2016 guidelines. Our practice was lacking for an objective assessment of the disease severity and activity. We think this is lacking for most routine day to day work clinical work. Our AS patients' characteristic did not differ much from the local and international published figures. Management wise, also there was no difference from others. However, we have much higher positive latent tuberculosis, pointing to the importance of such screening pre-biological treatment in the Middle East.

\section{Conclusion}

Our study has the advantage of good sample size and addressing practical aspects of AS management. However, it has the limitations of being retrospective. It revealed the lack of documentation of many important aspects concerning AS management. It showed that disease activity and severity assessment has not carried out in the majority of our patients. It also highlighted the importance of doing the tuberculosis pre-biologic screening in the Middle East, as almost a quarter of our patients were found to have latent Tuberculosis and who needed to be treated before the start of the biologics. It is worth mentioning and taking into consideration that in Qatar the population is heterogeneous, and it mainly includes many other Arabs and Asians, which accounts for more than $3 / 4$ of the population. However, from the point of the audit of view, this should not affect our results.

\section{Implications}

Our study highlighted the discrepancies between what is evidenced based and what was the real-life scenarios in managing our patients with AS.

\section{Acknowledgements}

To all our consultants in the rheumatology section at Hamad Medical Institute.

\section{Conflict of Interest}

None.

\section{Funding}

None.

\section{Availability of Data and Material}

Available on a secure computer.

\section{Authors' Contributions}

AWA has the idea, managed the progress of it at various stages, fully wrote the manuscript, YMY collected the data and analyzed under the supervision of AWA.

\section{References}

1. Zochling J, Braun J (2005) Management and treatment of ankylosing spondylitis. Curr Opin Rheumatol., 17 (4): 418-425.

2. John H Klippel, John H Stone, Leslie J Crofford, Cornelia M Weyand (2001) Primer on the Rheumatic Diseases: 250.

3. Braun J, Sieper J (2007) Ankylosing spondylitis. Lancet 369(9570): 1379-1390.

4. Carron P, Van Praet L, Van den Bosch F (2012) Peripheral manifestations in spondyloarthritis: relevance for diagnosis, classification and followup. Curr Opin Rheumatol 24 (4): 370-374.

5. Stolwijk C, Essers I, Van Tubergen A, Boonen A, Bazelier MT, et al. (2015) The epidemiology of extra-articular manifestations in ankylosing spondylitis: a population-based matched cohort study. Ann Rheum Dis 74 (7): 1373-1378.

6. Sieper J, Braun J, Rudwaleit M, Boonen A, Zink A (2002) Ankylosing spondylitis: an overview. Ann Rheum Dis 61 Suppl 3: iii8-18.

7. Otom A, Al-Ahmar MR (2013) HLA-B27 and ankylosing spondylitis in Jordan. GARJMMS 2 (12): 252-255.

8. Al-Arfaj A (1996) Profile of ankylosing spondylitis in Saudi Arabia. Clin Rheumatol 15(3): 287-289.

9. Tayel MY, Soliman E, El Baz WF, El Labaan A, Hamaad Y, et al. (2012) Registry of the clinical characteristics of spondyloarthritis in a cohort of the Egyptian population Rheumatol Int 32 (9): 2837-2842.

10. Fiaz Alam, Abdo Qaid Lutf, Nabeel Abdulla, Eman Hassan Satti, Mohammed Hammoudeh (2017) Characteristics of Ankylosing Spondylitis patients in Qatar. The Egyptian Rheumatologist 39(2): 103108.

11. D van der Heijde, S Ramiro, R Landewe, Xenofon Baraliakos, Filip Van den Bosch, et al. (2017) 2016 update of the ASAS-EULAR management 
recommendations for axial spondyloarthritis. Annals of the Rheumatic Disease 76: 978-991.

12. Alamanos, Papadopoulos NG, Voulgari PV, Karakatsanis A, Siozos C, et al. (2004)Epidemiology of ankylosing spondylitis in Northwest Greece, 1983-2002. Rheumatology (Oxford) 43 (5): 615-618.

13. Sampaio-Barros PD, Bertolo MB, Kraemer MH, Neto JF, Samara AM (2001) Primary Ankylosing Spondylitis: patterns of disease in a Brazilian population of 147 Patients J Rheumatol 28 (3): 560-565.

14. El Mansouri L, Bahiri R, Abourazzak FE, Abouqal R, Hajjaj-Hassouni N (2009) Two distinct patterns of ankylosing spondylitis in Moroccan patients Rheumatol Int 29 (12): 1423-1429.

15. Nazarinia MA, Ghaffarpasand F, Heiran HR, Habibagahi Z (2009) The pattern of ankylosing spondylitis in an Iranian population of 98 patients. Mod Rheumatol 19 (3): 309-315.

16. Stolwijk C, Van Tubergen A, Castillo-Ortiz JD, Boonen A (2015) Prevalence of extra-articular manifestations in patients with ankylosing spondylitis: a systematic review and meta-analysis. Ann Rheum Dis 74 (1): 65-73.

ISSN: 2574-1241

DOI: $10.26717 /$ BJSTR.2021.36.005904

Abdul-Wahab Al-Allaf. Biomed J Sci \& Tech Res

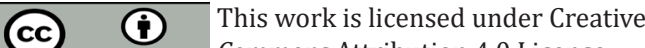
Commons Attribution 4.0 License

Submission Link: https://biomedres.us/submit-manuscript.php
17. Al-Amayreh IA, Zaidat BO (2000) Ankylosing spondylitis in Northern Jordan. Saudi Med J 21 (10): 950-952.

18. Uppal SS, Abraham M, Chowdhury RI, Kumari R, Pathan EM, et al. (2006) Ankylosing spondylitis and undifferentiated spondyloarthritis in Kuwait: a comparison between Arabs and South Asians. Clin Rheumatol 25 (2): 219-224.

19. Braun J, Sieper J (2007) Ankylosing spondylitis. Lancet 369(9570): 1379-1390.

20. Khan MA, Mathieu A, Sorrentino R, Akkoc N (2007) The pathogenetic role of HLA-B27 and its subtypes. Autoimmun Rev 6(3): 183-189.

21. Feldtkeller E, Khan MA, Van der Heijde D, Van der Linden S, Braun J (2003) Age at disease onset and diagnosis delay in HLA-B27 negative vs. positive patients with ankylosing spondylitis. Rheumatol Int 23(2): 61-66.

22. Frances Rees, Gillian Peffers, Carolyn Bell, Karen Obrenovic, Ravinder Sandhu, et al. (2012) Compliance with NICE guidance on the use of antiTNF agents in ankylosing spondylitis. Clin Med 12(6): 603-603. 
